\title{
VIDA PARA RUTH (1962) DE BASIL DEARDEN Y EL VEREDICTO (2017) DE RICHARD EYRE. LOS TESTIGOS DE JEHOVÁ Y LAS TRANSFUSIONES DE SANGRE: DOS VISIONES CINEMATOGRÁFICAS DEL MISMO DILEMA
}

\section{Life for Ruth (1962) by Basil Dearden and The Children Act (2017) by Richard Eyre. Jehova's Witnesses and blood transfusions: two cinematographic visions of the same dilemma}

\author{
Miguel ABAD VILA \\ Centro de Saúde Novoa Santos. Ourense (España). \\ Correo electrónico: mabadvila@gmail.com
}

Recibido: 24 de febrero de 2021

Aceptación: 23 de marzo de 2021

\section{Resumen}

El rechazo de las transfusiones de sangre por parte de los Testigos de Jehová representa un ejemplo clásico en el ámbito de la Bioética y la relación médico-paciente, cuando entran en colisión las intenciones y actuaciones de los médicos y las instituciones sanitarias, que tratan de salvaguardar la salud del paciente, con el necesario respeto de las decisiones personales del mismo respecto a las medidas terapéuticas que pudiera necesitar. Conceptos como la debida información, la confidencialidad, la libertad y la ausencia de toda coacción cobran entonces una especial importancia.

En este aspecto, presentamos dos visiones cinematográficas sobre el mismo dilema: qué hacer cuando un Testigo de Jehová rechaza una transfusión sanguínea, poniendo en serio peligro su propia vida. La primera de ellas es Vida para Ruth (1962) de Basil Dearden, una película realizada apenas un par de décadas después de que las máximas instituciones de los Testigos de Jehová adoptaran la firme decisión de rechazar este tratamiento. La segunda ella, tras el adecuado sosiego dogmático, nos la proporciona El veredicto (2017) de Richard Eyre, donde una juez especializada en asuntos familiares debe sentenciar respecto a unos padres, Testigos de 
VIDA PARA RUTH (1962) DE BASIL DEARDEN Y EL VEREDICTO (2017) DE RICHARD EYRE. LOS TESTIGOS DE JEHOVÁ Y LAS TRANSFUSIONES DE SANGRE

MIGUEL ABAD VILA

Jehová, que deniegan una transfusión de sangre a su propio hijo, menor de edad y gravemente enfermo.

Palabras clave: transfusiones de sangre; Testigos de Jehová; bioética.

\section{Summary}

The rejection of blood transfusions by Jehova's Withnesses represents a classic example in the field of Bioethics and doctor-patients relationship, where the intentions and actions of doctors and health institutions collide, trying to safeguard the healthy of the patient with the necessary respect for his personal decisions regarding the therapeutic measures he mat need. Concepts such as proper information, confidentiality, freedom and the absence of any coercion are especially important.

In this regard, we present two cinematographic visions on the same dilemma: what to do when a Jehova's Witness refuses a blood transfusion, putting his own life in serious danger. The first of them is Life for Ruth (1962) by Basil Dearden, a film made just a couple of decades after the highest institutions of Jehova's Witnesess made the firm decision to reject these therapeutic measures. The second one, with the appropriate dogmatic calm, provided by The Children Act (2017) by Richard Eyre, in which a specialized judge in family matters must sentence with respect to some parents, Jehova's Witnesses, who deny a blood transfusion to their own minor and seriously ill child.

Key words: blood transfusions; Jehova's Witnesses; bioethics.

\section{Introducción}

Charles Taze Russell (1852-1916) fue un líder religioso estadounidense que entre 1879 y 1880 fundó el movimiento Estudiantes de la Biblia, restauracionista, milenarista y antitrinitarista.

En 1881, el Pastor Russell fundó la Zion's Watch Tower Tract Society, con la finalidad de distribuir ejemplares de la Biblia, libros y revistas que difundieran sus doctrinas. En 1917, tras su fallecimiento, Joseph Franklin Rutherford (1869-1942) fue elegido nuevo presidente de la Watch Tower Bible and Tract Society of Pensilvania, la principal fuente de financiamiento y entidad jurídica más antigua de los testigos de Jehová. Pero sus desavenencias con otros líderes de la comunidad religiosa llevaron a su escisión en diferentes facciones. El grupo de Estudiantes de la Biblia que no se apartó de la Watch Tower de Rutherford, adoptaría en 1931 el nombre de Testigos de Jehová.

Hasta 1927, la Sociedad Watchtower entendía que las leyes bíblicas contra la sangre no eran vinculantes. El propio Russell aceptó que la prohibición recogida en el libro de los Hechos no era vinculante para los cristianos, y que solamente se respetó durante el primer siglo de nuestra era para evitar conflictos entre los conversos de origen judío y gentil. La vigencia de la Ley Mosaica, que proscribía también consumir carne de cerdo y determinados crustáceos, llegaba a su punto final con la muerte y resurrección de Jesucristo.

Pero en 1942, tras la defunción de Rutherford, Natan Homer Knorr (1905-1977) se convierte en su legatario, estableciendo la prohibición de las transfusiones de sangre. Precisamente en su publicación La Atalaya del 1 de julio de 1945, páginas 199 y 200, aparece la primera indicación de la prohibición de Dios sobre la sangre humana. Y aunque se incluía una mención a las transfusiones de sangre, éstas no fueron expresamente prohibidas $^{1}$.

Desde entonces, los Testigos de Jehová se han opuesto sistemáticamente a esta terapia aludiendo motivos religiosos, interpretando de una 
manera literal determinados mandatos divinos recogidos en las Sagradas Escrituras. Para ellos, tanto el Antiguo como el Nuevo Testamento conminan a la abstención de la sangre (Génesis 9:4; Levítico 17:10; Deuteronomio 12:23; Hechos $15: 28,29)$. Si no fuera suficiente, defienden que para Dios la sangre representa la vida (Levítico 17:14).

Históricamente la Wachtower ha ido modificando sus posicionamientos sobre el rechazo de las transfusiones de sangre. Antes de 1945, las transfusiones de sangre completa, así como de sus fracciones mayores (plasma, eritrocitos, leucocitos y plaquetas) y menores (albúmina, inmunoglobulinas, factores de coagulación, hemoglobina, hemina, interferones) era aceptada. Esto cambió radicalmente entre 1945 y 1982, cuando la transfusión de sangre o de cualquiera de sus fracciones fue vetada. Sin embargo, durante la década de 1980 la Watchtower revocó su posición sobre el rechazo de los trasplantes de órganos.

Entre 1982 y el 2000, se mantuvo la censura para las transfusiones de sangre completa y las fracciones mayores, pero comenzaron a permitirse algunas excepciones con los componentes menores. Hasta la actualidad, las transfusiones y los componentes mayores continúan prohibidos, pero ya se permite el uso terapéutico de las fracciones menores.

Debido a éstas y otras razones, durante décadas este grupo religioso ha suscitado el rechazo del resto de la sociedad, cuando no encarnizadas persecuciones e incluso campañas de exterminio, debido fundamentalmente a su objeción de conciencia ante el servicio militar, su insumisión ante cualquier símbolo patrio, como por ejemplo la bandera, y su oposición a los nacionalismos en general. Ejemplos de esto han sido su internamiento y ejecución en los campos de concentración nazis, donde fueron identificados mediante un triángulo morado invertido cosido en sus uniformes, o el hostigamiento de una pacífica comunidad de Testigos de Jehová en Beginning (2020) de Dea Kulumbegashvili, co-producción franco-georgiana galardonada con la Concha de Oro del prestigioso Festival de San Sebastián².

En 2017, la Corte Suprema rusa declaró organización extremista al Centro de Dirección de los Testigos de Jehová, prohibiendo su labor proselitista en todo su país ${ }^{3}$.

\section{El conflicto}

Desde la remota antigüedad, la sangre se consideraba un vigoroso determinante del vigor $y$ la salud. Algunas culturas promovieron su ingesta para reanimar a los enfermos, como la que procedía de las heridas de los gladiadores del circo romano, supuestamente un valioso tratamiento para la epilepsia.

Por ello no debería extrañarnos la siguiente historia. En 1492, cuando el Papa Inocencio VIII enfermó gravemente, sus asistentes decidieron administrarle sangre. Para ello recurrieron a tres niños de 10 años de edad, contratados por el capellán Buchard, con la siniestra intención de que donasen tan preciada fuente de vida al pontífice moribundo. La sangre le fue administrada por vía oral. Y aunque no se trató de una transfusión propiamente dicha, esta terapia sirvió para concienciar a la ciencia y a la sociedad sobre la necesidad de donar sangre de un individuo sano a otro enfermo para intentar salvarle la vida. Lástima que el denodado esfuerzo papal finalizara con la muerte de Inocencio VIII y la trágica pérdida de los tres pequeños donantes ${ }^{4}$.

A lo largo de su existencia, la teología cristiana ha reflexionado profusamente sobre la dignidad de la vida humana. Esta circunstancia no se ha restringido a cuestiones meramente antropológicas, sino que se fundamenta en la consideración de Dios como señor de la vida. Por lo tanto, se sitúa más allá de los límites puramente éticos, hasta remontarse a la misma consideración teológica de Dios y del propio ser humano ${ }^{5}$. 
Sin embargo, para los Testigos de Jehová las transfusiones de sangre constituyen una opción terapéutica inaceptable que colisiona completamente con sus creencias religiosas, hasta tal punto de preferir la muerte como alternativa. De hecho, constituyen el único grupo religioso que se opone frontalmente a esta terapia. Su interpretación literal de las escrituras considera que comer sangre es sinónimo de su absorción corporal. De ahí su oposición radical, aunque en algunos aspectos no desdeñables ha ido variando, y quizás se modifique, a lo largo del tiempo ${ }^{6}$.

Llegado el momento justificativo de la necesidad de una transfusión, el médico deberá enfrentarse a un dilema adicional en el tratamiento de estos pacientes, asegurándose en todo momento que cualquier tipo de decisión adoptada tenga en consideración los principios de información, confidencialidad, libertad y ausencia de coacciones.

Dentro del campo de la Bioética, existen posicionamientos a favor del respeto de los derechos y la autonomía del paciente, en este caso la de los testigos de Jehová, a la hora de demandar tratamientos médicos en los que no se empleen transfusiones de sangre ${ }^{7}$.

La Watch Tower Bible and Tract of Pennsylvania, entendiendo que la asistencia sanitaria de sus fieles puede suponer un desafío para la comunidad médica en determinadas ocasiones, edita periódicamente manuales con información destinada a los profesionales (médicos, jueces, asistentes sociales) que en algún momento deban

Vida para Ruth (1962) de Basil Dearden

Ficha técnica

Título: Una vida para Ruth. Título Original: Life for Ruth. País: Reino Unido. tomar decisiones para salvaguardar la salud de los testigos de Jehová o su descendencia8.

Estos manuales recogen información sobre la Red de Comités de Enlace con los Hospitales, formados por personas con formación y experiencia sanitaria, y que sirven de enlace con los médicos que consideren la necesidad de administrar una transfusión de sangre a un testigo de Jehová o a uno de sus hijos.

Asimismo, las congregaciones de testigos de Jehová disponen de los recursos necesarios para asegurarse que el enfermo o su familia se mantengan firmes a la hora de rechazar una transfusión sanguínea, aunque esta decisión pueda acarrear el fallecimiento del paciente. En este sentido, en El Veredicto (2017) de Richard Eyre, los facultativos se quejan de las frecuentes visitas que realizan a los fieles hospitalizados distintos asociados de su congregación, generalmente siervos ministeriales o miembros del cuerpo de ancianos o presbíteros, encargados de velar por la conducta de sus adeptos. Dentro de los Testigos de Jehová existen también comités judiciales encargados de amonestar y expulsar, llegado el caso, a todo aquel disidente de cuerpo doctrinal o a los que hayan cometido pecados muy graves.

La estructura de los testigos de Jehová es verticalista, desde las bases de los predicadores hasta las cúpulas superiores de las congregaciones: circuitos, distritos, sucursales, zonas y el denominado cuerpo gobernante, con su sede oficial (Watch Tower) en Brooklyn, Nueva York, formada por 12 personas 9 .

Año: 1962.

Director: Basil Dearden.

Música: William Alwyn.

Fotografía: Otto Heller.

Montaje: John D. Guthridge.

Guión: Janet Green, John McCormick. 
Intérpretes: Michael Craig (John Paul Harris), Patrick McGoohan (Doctor James «Jim» Brown), Janet Munro (Pat Harris), Paul Rogers (Counsel Hart Jacobs), Malcom Keen (Mr. Harris Sr.), Megs Jenkins (Mrs. Gordon), Michael Bryant (John's counsel), Leslie Sands (Clyde - newspaper editor), Norman Wooland (Counsel for the Crown), John Barrie (Mr. Gordon), Walter Hudd (Judge), Michael Aldridge (Dr. Richard Harvard), Basil Dignam (Lawyer Mapleton), Maureen Pryor (Teddy's mother), Kenneth J. Warren (Sergeant Finlay), Lynn Taylor (Ruth).

Color: blanco y negro.

Duración: 15 minutos.

Género: drama.

Idioma original: inglés.

Productora: Allied Film Makers (AFM).

Sinopsis: Cuando la pequeña Ruth, hija de John y Pat Harris, resulta gravemente herida en un accidente en el mar, los médicos les transmiten la necesidad de transfundirla urgentemente. Debido a sus creencias religiosas, los Harris no conceden el permiso y la pequeña muere. Una vez la policía exime al padre de toda negligencia, el médico a cargo del caso intenta presentar casos contra él, por homicidio.

\section{Enlaces}

Tráiler original

Situémonos en el tiempo. A comienzos del siglo XX, Karl Landsteiner (1868-1943) descubrió los grupos sanguíneos $A B O$, hazaña completada en 1907 con el del grupo $A B$ por el médico checo Jan Jansky (1873-1921). Sobre estos hallazgos podríamos situar el comienzo de la historia moderna de las transfusiones sanguíneas. Ese mismo año, el cirujano francés Alexis Carrel (1873-1944) promovió en Nueva York la transfusión directa de sangre entre un donante y un receptor mediante una

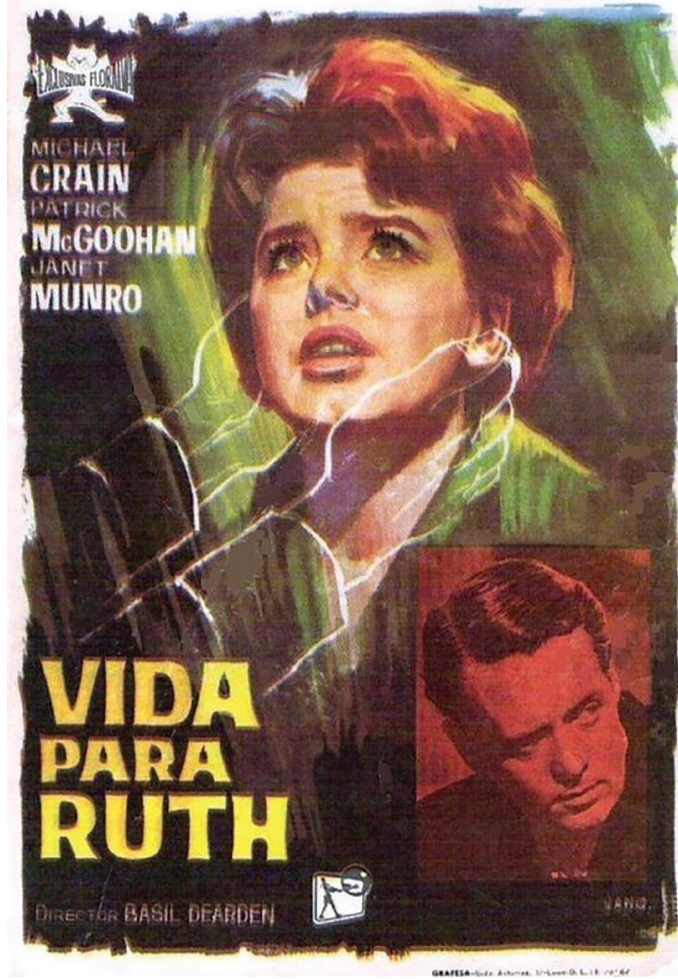

Cartel español.

"Su acción es criminal como el infierno; sus razones... inocentes, como el propio cielo»

anastomosis arterio-venosa. Y por sus descubrimientos recibió en 1912 el Premio Nobel de Medicina ${ }^{10}$.

Aún así, a pesar del avance constante de la ciencia y la técnica, la coagulación de la sangre constituía un serio problema en las transfusiones pioneras. La solución vino de la mano del investigador argentino Luis Agote (1868-1954), que, al intentar conservar la sangre necesaria para tratar a sus pacientes hemofílicos, descubrió que el citrato de sodio evitaba la formación de 
coágulos. El 9 de noviembre de 1914 documentó la primera transfusión exitosa entre humanos, empleando como anticoagulante la solución ACD (ácido cítrico, citrato sódico y dextrosa) ${ }^{11}$.

Finalmente, en 1937, el descubrimiento del factor Rh por Karl Landsteiner y Alexander S. Wiener (1907-1976), una proteína presente en los glóbulos rojos en aproximadamente el $85 \%$ de la población, vino a solucionar los problemas originados por la incompatibilidad en las transfusiones de sangre.

Pero la compatibilidad no fue el único obstáculo evitable. Demasiadas infecciones fueron transmitidas a través de las transfusiones: parasitarias (Plasmodium spp, Tripanosoma cruzi, Babesia microfti, Leishmania spp., Toxoplasma gondii), víricas (hepatitis $\mathrm{A}, \mathrm{B}, \mathrm{C}, \mathrm{VIH}$, citomegalovirus, Epstein-Barr, parvovirus B19, etc.), bacterianas (Staphylococcus aureus, Pseudomonas aeruginosa, Escherichia coli, Salmonella spp., Yersinia enterocolitica, Serratia marcescens, Treponema pallidum, Brucella spp., Borrelia burgdorferi, etc.) y otras (priones) ${ }^{12}$.

A pesar del implemento progresivo de las medidas de seguridad en los bancos de sangre, para garantizar la inocuidad de estos tratamientos, los accidentes transfusionales y la trasmisión de enfermedades por medio de la sangre transfundida eran conocidas en 1962, cuando se rodó la película de Basil Dearden, una realidad que entonces contribuía al rechazo de las transfusiones, y no solo por razones espirituales.

De todas las maneras, en el guión de esta película de tesis no existe ni una sola línea al respecto. Tampoco los testigos de Jehová son mencionados expresamente, si bien se entiende que el protagonista, el ingeniero de minas John Paul Harris (espléndido Michael Craig), profesa esta fe de manera incontestable. La negativa a que su hija Ruth (Lynn Taylor, en la realidad hija de un minero y futura maestra), pequeña moribunda de 8 años gravemente herida en un accidente en el mar, reciba una transfusión de sangre tiene motivos estrictamente religiosos. Las fatales consecuencias de semejante decisión desencadenarán un drama en el que destacan el sufrimiento de la joven esposa y madre, Pat Harris (Janet Munro), la tragedia familiar, el rechazo social, la avidez por el sensacionalismo periodístico, los dilemas éticos y la pugna judicial entre la acusación y la defensa, una vez el Doctor Brown (Patrick McGoohan) consigue llevar ante el juez (Walter Hudd) al tribulado padre, en un testarudo, incluso antipático y encarnizado empeño, destinado a impedir situaciones semejantes en otros escenarios y con otros protagonistas.

Esta producción de la AFM (Allied Film Makers), incomprensiblemente desconocida para el público general, evidencia el deber de los médicos a la hora de salvar la vida de los pacientes, irrenunciable incluso ante razones tan poderosas como la religión y la ideología. Pero esa titánica labor no debe descansar únicamente sobre sus hombros, ni siquiera sobre los de los padres que tienen que decidir sobre la vida o la muerte de sus hijos menores. Para ello resulta fundamental la salvaguarda de las leyes, como representación de los poderes públicos, preservando la integridad de aquellos más frágiles que pudieran resultar perjudicados por las decisiones de otros, aún viniendo de sus propios progenitores.

El mérito incuestionable de Vida para Ruth (1962) de Basil Dearden, rodada en los estudios de Pinewood y en escenarios naturales del condado de East Durham del Noreste de Inglaterra, es la admirable ecuanimidad con la que trata a todos los personajes, sin maniqueos sentimentalismos ${ }^{13}$. 


\section{El veredicto (2017) de Richard Eyre}

Ficha técnica

Título: El veredicto.

Título Original: The Children Act.

País: Reino Unido.

Año: 2017.

Director: Richard Eyre.

Música: Stephen Warbeck.

Fotografía: Andrew Dunn.

Montaje: Dan Farrell.

Guión: Ian McEwan.

Intérpretes: Fionn Withehead (Adam Henry), Emma Thompson (Fiona Maye), Stanley Tucci (Jack Maye), Jason Watkins (Nigel Pauling), Ben Chaplin (Kevin Henry), Eileen Walsh (Naomi Henry), Nikki Amuka-Bird (Amadia Kalu QC), Rosie Cavaliero (Marina Green), Rupert Vansittart (Sherwood Runcie), Anthony Calf (Mark Berner), Hicholas Jones (Professor Rodney Carter), Chris Wilson (Barrister), Andrew Havill (George), Paul Jesson (Humphrey), Naomi Frederick (Amanda).

Color: color.

Duración: 105 minutos.

Género: drama.

Idioma original: inglés.

Productoras: Toledo Productions, BBC Films, FilmNation Entertainment.

Sinopsis: Fiona Maye es una prestigiosa jueza del Tribunal Superior de Londres, especializada en Derecho Familiar. Mientras se encuentra atravesando una grave crisis matrimonial, a su juzgado llega el caso del joven Adam Henry, un adolescente con leucemia que se niega a recibir una transfusión de sangre al ser Testigo de Jehová. La jueza

Medio siglo después, el cine británico se aproximaría nuevamente al dilema transfusiones y los testigos de Jehová, que en esta ocasión son mencionados de forma explícita.
Maye deberá enfrentarse a sus sentimientos ocultos mientras trata de convencer a Adam para que acepte el tratamiento y evite la muerte.

\section{Enlaces}

Tráiler original

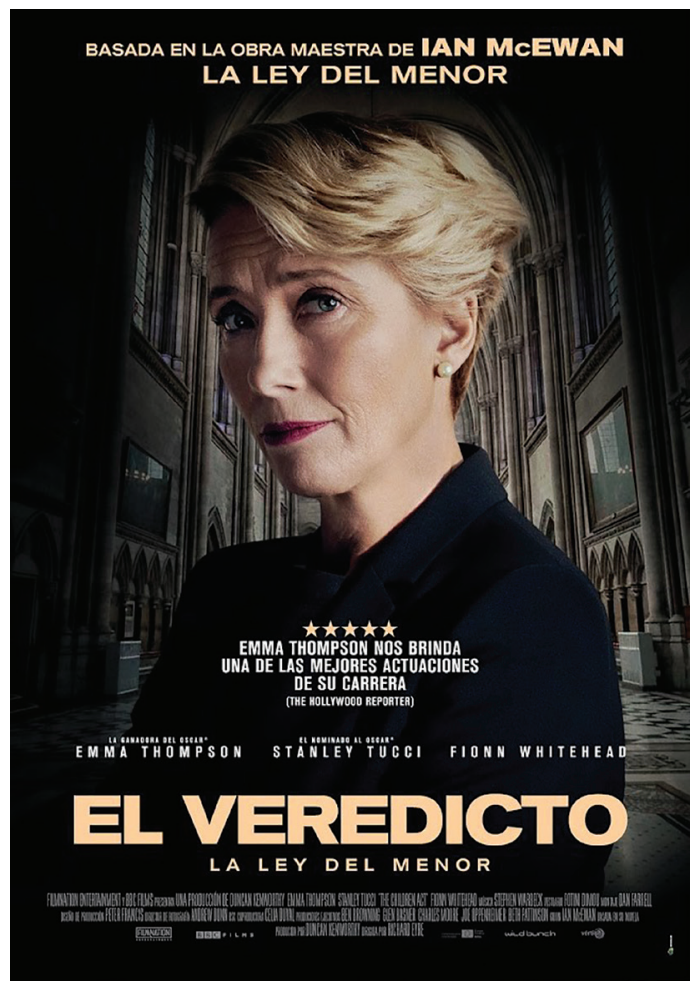

Cartel español.

- "¿Por qué la sangre es tan importante para

Dios. Por qué Dios es tan insistente?

- Porque es donde está el alma, justo en la

sangre. $Y$ por eso le pertenece a él.

- Muy bien dicho: el alma, la vida, están en la sangre. Y no son nuestras. Son de Dios»... 
Henry (Fionn Whitehead), enfermo de leucemia y subsidiario de una transfusión que le permita sobrevivir a la quimioterapia.

El veredicto (2017) de Richard Eyre es una película sobre el difícil equilibrio, cuando no imposible, entre las decisiones judiciales y sus consecuencias en el terreno de lo personal, lo emocional y lo moral ${ }^{14}$.

De la humilde vivienda de los Harris, en un barrio obrero de la cuenca minera de las Cullercoats, con sus calles cubiertas por nieve sucia y deshilachada, y sus escarpadas costas bañadas por las procelosas aguas del Mar del Norte, pasamos a los lujosos apartamentos de Field Court, la acomodada residencia de la jueza Maye y su esposo Jack (Stanley Tucci), con su valioso piano Fazoli incluido, en el centro de Londres y cercana a los Reales Tribunales, donde su señoría trabaja impartiendo justicia en conflictos familiares.

Los padres de Adam, Kevin (Ben Chaplin) y Naomi Henry (Eileen Walsh) se niegan por razones religiosas a que los médicos suministren una transfusión a su hijo de 17 años, menor de edad apenas por dos meses. Pero en esta ocasión, el equipo médico dirigido por el jefe de Hematología, el Profesor Rodney Carter (Nicholas Jones), respetando previamente los principios de la ética médica (información, confidencialidad, libertad y ausencia de coacciones) ha recabado el dictamen de la justicia y el caso ha recaído en el tribunal de la jueza Maye ${ }^{15}$.

Respecto a la fidelidad divina y la sumisión de los menores al mandato que rechaza las transfusiones de sangre, en 1994 la portada de la revista iDespertad! mostraba el titular Jóvenes que ponen a Dios en primer lugar, recogiendo los testimonios de cinco jóvenes de entre 12 y 17 años que se habían negado a recibir transfusiones de sangre antes de abandonar sus creencias. Dos de ellos fallecieron a causa de neoplasias hematológicas ${ }^{16}$.

Adicionalmente, en este film la defensa de los Henry alega causas médicas para oponerse a la decisión facultativa, como por ejemplo el riesgo de contagio de enfermedades infecciosas (VIH - SIDA, hepatitis, enfermedad de Lyme, enfermedad de Chagas, sífilis), la lesión pulmonar aguda producida por transfusión (TRALI - transfusión related acute lug injury) o la transmisión de la variante de la enfermedad de Creutzfeldt-Jakob.

El prestigioso abogado Mark Berner (Anthony Calf) es contratado por el hospital para tratar de conseguir el permiso legal para la transfusión sanguínea. Además de su relación profesional, Berner y Fiona Maye conforman un dúo musical que actúa en celebraciones y ocasiones especiales. Mediante su acerado alegato, Berner intenta demostrar el comportamiento sectario de los testigos de Jehová, que consideran la homosexualidad y la masturbación como pecados. También por aceptar al pie de la letra unos mandatos escritos en la Edad de Hierro, cuando no existían las transfusiones, tamizados por un comité de estudiosos de la Biblia reunido en Brooklyn (Nueva York) en 1945. Este dato está confirmado por los propios testigos de Jehová, que tras la II Guerra Mundial y la generalización de las transfusiones, creyeron entender que estaban contra la ley de $\operatorname{Dios}^{17}$.

Además, el abogado Berner (Anthony Calf) interroga a los Henry sobre la posibilidad de que Adam pudiera ser expulsado de su congregación si aceptase una transfusión de sangre, posibilidad que al parecer está contemplada entre los testigos de Jehová desde 1961.

Después de entrevistarse con el joven en el hospital, la jueza Maye autoriza la transfusión, al considerar que el derecho a la vida está por encima del derecho a la dignidad. Antes de tomar esta decisión, contemplamos a la magistrada en una escena en su despacho consultando un artículo sobre la asistencia médica específica para los testigos de Jehová: Assisting Patients who refuse Transfusion, de Paul M. Stevenson, director del Comité Hospitalario de Enlace con los testigos de Jehová de Exeter (Reino Unido). En la realidad, 
este autor ha escrito otras publicaciones al respecto ${ }^{18}$.

Una vez recuperado, Adam (Fionn Whitehead) desarrolla un inusitado y repentino ansia vital que le lleva a apartarse de las creencias familiares y de las reuniones del Salón del Reino de los testigos de Jehová junto a sus padres, fascinado por la personalidad de la jueza, a su vez inmersa en una profunda crítica matrimonial y existencial.

Con un exquisito cuidado, el cineasta británico se había aproximado previamente a la enfermedad y la muerte en Iris (2001) de Richard Eyre, la perdurable historia de amor entre la brillante filósofa y escritora Iris Murdoch (Judi Dench), abatida por el Alzheimer, y su esposo John Bayley (Jim Broadbent, ganador de un Oscar por esta interpretación).

Para esta ocasión, contó como guionista con el reconocido escritor lan McEwan, considerado una de las personas más influyentes de la cultura británica, por otra parte, el autor de la novela original. En The Children Act (2014) de lan McEwan se reflexiona sobre las dificultades y los sentimientos de una jueza del Tribunal Superior de Justicia cuando debe decidir sobre asuntos de vida o muerte, como por ejemplo la separación de dos hermanos siameses a costa de la vida de uno de los dos pequeños, o la necesidad de transfundir a un adolescente testigo de Jehová con leucemia. El conflicto entre ley y religión constituye el asunto central de la novela y la película.

Ian McEwan tiene fama de riguroso y objetivo. Para escribir sus libros suele documentarse previamente de manera concienzuda. Los casos de El veredicto (2017) de Richard Eyre están basados en ciertos precedentes legales juzgados en los tribunales británicos durante las dos últimas décadas. Y aunque McEwan tampoco los ha mencionado, se trataba de sentencias emitidas por los jueces Sir Alan Ward y Sir James Munby. Para el escritor, si deseamos discutir sobre el conflicto entre los derechos legales y los deberes religiosos, especialmente cuando surgen en el contexto ético médico-legal, resultaría esencial la lectura de las sentencias originales, basadas en concienzudos y cuidados razonamientos, y que para McEwan constituyen por derecho propio auténticas obras de arte y ciencia ${ }^{19}$.

\section{Los Testigos de Jehová y el cine}

Desde los primeros años del pasado siglo, los responsables de la Watch Tower se percataron del valor del cine para difundir la doctrina de los Testigos de Jehová. Su primera producción fue Foto-Drama de la Creación (1914) de Charles Taze Russell, 8 horas de duración divididas en 4 partes que incorporaban sonido sincronizado (un fondo de música clásica), escenas en movimiento y diapositivas en color. Se estrenó en Nueva York, el 11 de enero de 1914.

Esta obra contenía 96 breves sermones bíblicos leídos por una de las voces masculinas más conocidas de la época. La mayor parte de las secuencias y las diapositivas en vidrio fueron realizadas en estudios de Filadelfia, Nueva York, París y Londres. Artistas profesionales pintaron a mano las diapositivas y las imágenes de la película, especialmente el Estudio de Arte de Betel. Algunos de sus miembros actuaron como improvisados actores en la ciudad de Yonkers (Nueva York), interpretando los personajes de Abraham, Isaac y el ángel que impidió su sacrificio ${ }^{20}$.

Entre 1914 y 1918, el Foto-Drama de la Creación (1914) de Charles Taze Russell se difundió gratuitamente por los Estados Unidos. Asimismo, en 1916 se realizó una versión reducida, el Drama Eureka, para su más amplia distribución.

En 1916, tras la defunción de Russell, empujados por el déficit económico que suponía el inmenso esfuerzo de las proyecciones gratuitas, varios adeptos decidieron comprar esta obra a la Sociedad, cuyos derechos de exhibición finalizaban el 31 de marzo de 1917. A partir de entonces, éstos pasaron a la Mena Film Company, con la condición de que una copia del Foto-Drama 
quedaría en posesión de la Sociedad para la difusión interna en sus instalaciones.

Este contrato no llegó a prosperar. En poco tiempo, muchos adeptos de diferentes partes exigieron a la Sociedad que no vendiera el FotoDrama y que recuperara el control sobre el mismo. A pesar de esta desvinculación, la Mena Film Company continuó su trayectoria independiente, produciendo en 1918 una película llamada Restitution (1918) de Howard Gaye, sin el soporte económico de la Sociedad pero contando en todo momento con su apoyo explícito. En un principio este filme iba a proyectarse comercialmente. Pero tras su lanzamiento en 1918, en una asamblea de los Estudiantes de la Biblia en Seattle, los sucesivos cismas dentro de la Asociación Internacional de Estudiantes de la Biblia (IBSA) provocaron que nunca llegara a exhibirse en las salas de cine.

Restitution (1914) de Charles Taze Russell fue reestrenada como The Conquering Christ. A finales de los años 20, Leslie Jones, un antiguo director de la Mena Film Company, comenzó a vender películas de $16 \mathrm{~mm}$ en segmentos de 7 minutos de duración en forma de una serie, bajo el nombre de Redención, si bien la mayoría de ellos extraviados en la actualidad; solamente uno de ellos ha sido recuperado recientemente.

A finales de 1922, la Watch Tower ofreció a sus suscriptores 3 películas cortas acompañadas de un proyector casero de la compañía Kimeno, por el precio de 65 dólares. Dicha distribución apena duró 1 año, debido a la incapacidad de Kimeno para satisfacer todos los pedidos solicitados. En las 3 películas aparecía John Franklin Rutherford, el sucesor de Charles Taze Russell.

Estas películas fueron proyectadas entre el 5 y el 13 de septiembre de 1922, en la asamblea de los Estudiantes de la Biblia celebrada en Cedar Point (Ohio). Un equipo profesional de cine se encargó de filmar además un documental de dicha reunión, ofrecido en octubre de 1922 por el periódico New Era Enterprise.
Desafortunadamente, todo este material se perdió definitivamente. Ni siquiera existen copias en la sede mundial de los Testigos de Jehová. Solamente se conoce su existencia por referencias escritas de la época.

En 1954, la Watch Tower produjo una nueva película de cine mudo, en formato $16 \mathrm{~mm}$, una demostración de su funcionamiento general y de la magnitud de los Testigos de Jehová a nivel mundial. En 1956, 1959 y 1964 produjeron nuevas películas en $16 \mathrm{~mm}$, utilizadas como material de difusión doctrinal. Años más tarde, la Watchtower Bible \& Tract Society of New York realizó 2 producciones más: Dios no puede mentir (1966) y Heritage (1966). Ésta última fue empleada para contrastar el estilo de vida mundano, especialmente de la juventud, en contraposición al de aquellos que eligieron regirse por los principios bíblicos.

\section{Cirugía sin sangre}

Como podemos comprobar en $\mathrm{El}$ veredicto (2017) de Richard Eyre, las transfusiones de sangre continúan provocando el rechazo de los Testigos de Jehová, y no solamente por cuestiones estrictamente religiosas. En la película sus abogados tratan de exponer ante la jueza Maye (Emma Thompson) las diferentes patologías que pueden ocasionar: infecciones, reacciones hemolíticas, reacciones alérgicas y otras complicaciones menos frecuentes, como la enfermedad de injerto contra huésped, en la que los glóbulos blancos transfundidos atacan la médula ósea del trasfundido.

Los primeros casos de infecciones virales transmitidos por las transfusiones fueron reportados en 1943. Los estudios de laboratorio para cribar la sangre y evitar dichas infecciones se iniciaron en 1969, con la identificación del antígeno de superficie de la hepatitis $B^{21}$.

Desde entonces y hasta la actualidad, los bancos de sangre continúan trabajando en las 
condiciones técnicas más estrictas para garantizar la seguridad de las transfusiones. Aún así, los especialistas de las áreas quirúrgicas y de anestesia y reanimación llevan años trabajando en las denominadas técnicas de ahorro de sangre, minimizando el uso de las transfusiones a situaciones estrictamente necesarias. Quizás la influencia de los Testigos de Jehová allá tenido algo de influencia en ello.

La visión bioética de tales cuestiones exige armonizar los valores individuales del paciente con los principios de autonomía, justicia, beneficencia y no maleficencia. En el caso de los testigos de Jehová respetar su autonomía significa no solamente preservar su vida, sino tener en cuenta cómo esa persona debe vivir realmente su vida ${ }^{22}$.

Aunque el dilema testigo de Jehová - transfusión es un clásico, incluso cinematográfico, el desarrollo de técnicas para el ahorro de sangre va más allá de las creencias religiosas, llegando a convertirse en una recomendación de la Organización Mundial de la Salud (OMS), que desde 2010 ha apostado por estos métodos, al constatar que las transfusiones alogénicas representan un recurso limitado, caro y no exento de riesgos y efectos adversos.

Se basan en razones fundamentales, que permiten ahorrar sangre, evitar los accidentes transfusionales y satisfacer la demanda creciente de este tipo de operaciones, por razones religiosas (como en el caso de los testigos de Jehová), y también por razones particulares e inmunológicas. Diferentes hospitales públicos y privados españoles disponen ya de unidades sin sangre y el tipo de intervenciones quirúrgicas que pueden realizarse cada día es mayor.

Los programas de gestión de la sangre del propio paciente (PMB por sus siglas en inglés: Patient Blood Management) constituyen una de estas alternativas, así como el rescate celular, en el que una máquina extrae la sangre de la intervención quirúrgica, la depura y la repone en el mismo paciente. Pero para que un testigo de Jehová la acepte, deberá garantizarse que el flujo de sangre no queda interrumpido en ningún momento de este proceso.

La vasoconstricción, física o química, es otra técnica empleada por los cirujanos para ahorrar sangre, minimizando el riesgo de hemorragia y la necesidad de una transfusión de sangre. En este sentido, la anestesia tumescente, mediante una mezcla muy diluida de lidocaína y una pequeña cantidad de adrenalina, favorece la vasoconstricción y evita las hemorragias.

Otra alternativa aceptada por los testigos de Jehová es la eritropoyetina, que estimula producción de células sanguíneas. En lugar de una transfusión, al paciente se le administran fluidos intravenosos y este fármaco, aunque su efecto no es inmediato.

El tratamiento con fracciones sanguíneas menores también puede representar una alternativa a las transfusiones en estos pacientes, es decir, productos derivados de la sangre como plasma, plaquetas o glóbulos blancos y rojos. $Y$ si bien por el momento sus dirigentes no se han manifestado expresamente en contra, porque la Biblia no da detalles al respecto, descargan sobre la conciencia de cada adepto su decisión consciente ante $\operatorname{Dios}^{23}$.

\section{Referencias}

1. Murillo-Godinez G. Las transfusiones de sangre y los Testigos de Jehová. Aspectos ético-médico-legales aún no resueltos. Med. Int. Mex. 2010; 26 (4): 390-396

2. Medina M. «Begining»: la ganadora de San Sebastián no deja indiferente a nadie. El confidencial [Internet] 4 de diciembre de 2020

3. Redacción. BBC News Mundo [Internet]. ¿Por qué Rusia prohibió a los Testigos de Jehová? 21 de abril de 2017.

4. Marrón-Peña M. Historia de la transfusión sanguínea. Rev. Mex. Anestesiol. 2017; 40 (3) 233-238.

5. Flecha J.R. La Fuente de la vida: Manual de Bioética. Salamanca: Ed. Sígueme; 2000. p 34-35. 


\section{VIDA PARA RUTH (1962) DE BASIL DEARDEN Y EL VEREDICTO (2017) DE RICHARD EYRE. LOS TESTIGOS DE JEHOVÁ Y LAS TRANSFUSIONES DE SANGRE}

MIGUEL ABAD VILA

6. Grundy P. Los Testigos de Jehová y las transfusiones de sangre. Verdad TJ.com [Internet].

7. Malyon D. Transfusion-free treatment of Jehova's Witnesses: respecting tha autonomous patient's rights. J. Med. Ethics. 1998;24(5):302-307.

8. Watchtower Bible and Tract Society of New York, INC. International Bible Students Association. La Familia, su cuidado y protección: tratamiento médico para testigos de Jehová. 1995, Brooklyn - Nueva York.

9. Calzato WA. Testigos de Jehová. Mundo, creencias, conducta. Gazeta de Antropología. 2006; (25): 1-12.

10. Izaguirre AR, De Michelli A. En torno a la Historia de las Transfusiones Sanguíneas. Rev. Invest. Clin. 2002; 54(6): 552-558

11. Agüero AL, Damián A. Luis Agote y su aporte a la ciencia universal. Rev Argent Salud Pública, 2019; 10 (38): 43-46.

12. Sánchez Frenes P. Las enfermedades infecciosas y la transfusión de sangre. 2012 Rev. Latinoamer. Patol. Clin. 59(4): 186-193.

13. García de Fórmica-Corsi JM. Vida para Ruth: cine de denuncia admirablemente ecuánime. La mano del extranjero [Internet]. 24 de junio de 2016.

14. Ocaña J. Reglas humanas, normas divinas. El País [Internet]. 23 de noviembre de 2018.
15. Besio M, Besio F. Testigos de Jehová y transfusión sanguínea. . Reflexión desde una ética natural. Rev. Chil. Obstet. Ginecol. 2006; 71 (4): 274-279.

16. Chryssides GD. Why Opposition? An Explanations of Hostility Towards Jehova's Witnesses. The Journal of CESNUR. 2021, 5/1: 16-38.

17. La Atalaya. Edición de estudio [Internet]. 15 de junio de2000. Preguntas de los lectores.

18. Gordon Melton J. Opposition to Jehovah's Witnesses in the United States Through the Twentieth Century. The Journal of CESNUR. 2021, 5/1: 39-53.

19. Paul M. The Children Act - a novel approach. [Internet]. 8 de octubre de 2014. The Transparency Project.

20. Testigos de Jehová. De nuestros Archivos. El «FotoDrama» cumple 100 años [Internet]. Febrero 2014.

21. Rojo Medina J. Enfermedades infecciosas transmitidas por transmisión. Panorama internacional y en México. Gaceta Med. Mex. 2014; 150: 78-83.

22. Retamales A. Autonomía del paciente: los testigos de Jehová y la elección de alternativas a la transfusión. Rev. Chil. Obstet. Ginecol. 2006; 71 (4): 280-287.

23. La Atalaya. Anunciando el Reino de Jehová. Preguntas de los lectores 2004 [Internet].

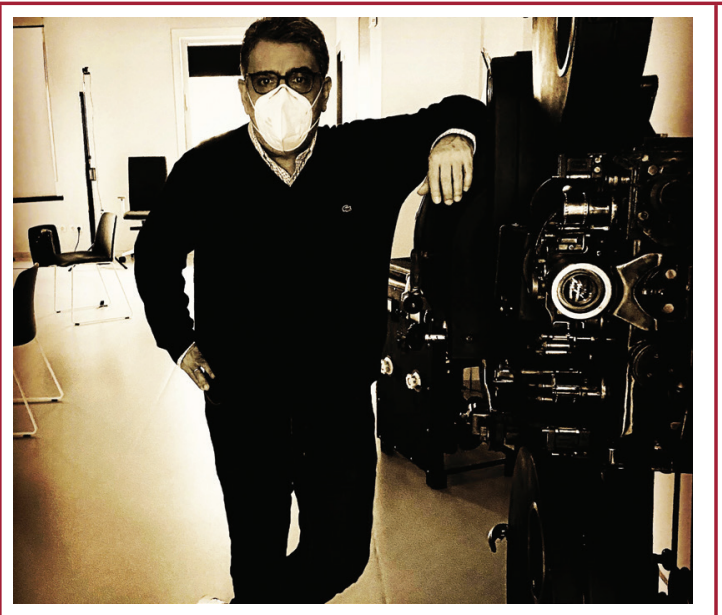

Licenciado en Medicina por la Universidad de Santiago de Compostela. Doctor en Medicina por la Universidad de Santiago de Compostela. Diplomado en Salud Pública. Máster en Gestión Sanitaria por la Universidad de Vigo. Máster en Derecho Sanitario por la Universidad de Castilla - La Mancha. Médico de Familia en el Centro de Saúde «Novoa Santos», Servizo Galego e Saúde, Ourense. Colaborador habitual de "La Región» (Ourense), "Atlántico Diario» (Vigo) y «Onda Cero" (Ourense). Autor de los blogs "Cartas de Aloysius» y "MEDYCINE». 